\title{
Activity Limitations of Patients with Stroke Attending Out-patient Facilities in the Western Cape, South Africa
}

\begin{abstract}
A stroke can affect individuals at the levels of impairment, activity and participation. The aim of this study is to determine the activity limitations of stroke patients receiving rehabilitation at out-patient Community Health Centres (CHCs). A longitudinal observational study was conducted. Activity limitations were measured using the Rivermead Motor Assessment Scale, the Barthel Index and the Nottingham Extended Activities of Daily living scale (NEADL). The study population consisted of 100 patients with stroke consecutively admitted to the Community Health Centres for therapy. The data was analysed using inferential and descriptive statistics. The majority of the participants were not able to climb stairs, travel on public transport, wash dishes, do washing, do household chores and shopping, socialize and manage the garden at six months post stroke. A significant improvement was noted in the ability of the participants to perform basic activities of daily living between baseline and two months, but not between two and six months. There was however a significant improvement in their ability to perform instrumental activities of daily living between two and six months. Although the participants were able to perform basic activities of daily living enabling them to be independent in their homes, they were unable to perform instrumental activities of daily living which limited their functioning in the community. There is a need for therapists to include activities which could facilitate re-integration into the community in their rehabilitation of patients with stroke.
\end{abstract}

\section{KEY WORDS: ACTIVITY LIMITATIONS, STROKE, OUT-PATIENT FACILITIES}

\section{INTRODUCTION}

Stroke is a major cause of death and disability worldwide (Feigin, et al 2003). Disability post-stroke can be conceptualised using the International Classification of Functioning, Disability and Health (ICF) as described by the World Health Organisation (2001). The ICF assists in analysing the patient's problems post stroke as well as enabling a systematic analysis of rehabilitation interventions (Wade and de Jong 2000).

A stroke can affect individuals at the levels of impairment, activity and participation. The activity limitations experienced post-stroke include basic and instrumental activities of daily living. Basic activities of daily living include

\section{Corresponding Author:}

Prof A Rhoda

Department of Physiotherapy

University of the Western Cape

Bellville 7535

Western Cape, South Africa

Email: arhoda@uwc.ac.za bathing, eating, dressing, toileting, bladder and bowel control, mobility, transfers and the ability to climb stairs (Mayo et al 1999). Instrumental activities are those activities needed for people to function within their communities and to run their households. The most common instrumental activities that patients with stroke are not able to perform post-incident include washing clothes, shopping and house work (Hartman-Maeir et al 2007; Rouillard 2006), as well as travelling by public transport (Rouillard 2006).

Studies conducted in both developed and developing countries have revealed that patients with stroke need assistance with activities of daily living. In a population-based study conducted in New Zealand, Bonita et al (1997) found that one third of the stroke population required assistance with at least one activity of daily living with a gender differential. In studies conducted in sub-Saharan Africa, patients with stroke appeared to be more dependent on others for self-care (SASPI Project Team 2004; Walker et al 2000). Walker et al (2000) reported that in Tanzania the number of people needing assistance with at least one activity of daily living was $60 \%$. In a South African study, 66\% of stroke survivors needed assistance (SASPI Project Team 2004).

Stroke is one of the most common chronic lifestyle diseases in the Western Cape, South Africa (Bradshaw et al., 2004). This disease places a high burden on patients, their families, the communities in which they live, as well as the health care system and the state. The majority of patients with stroke are referred to out-patient facilities for rehabilitation even in the acute stages (Rhoda and Hendry 2006). Studies have indicated that an extended programme in the community, provided by a dedicated team could result in patients being discharged earlier from hospital (Anderson et al 2000; BautzHolter et al 2002; Donnelly et al 2004; Indredavik et al 2000; Mayo et al 2000). This approached was developed as the home setting, is conducive to relearning skills post stroke (Anderson et al 2000; 
Bautz-Holter et al 2002; Donnelly et al 2004; Indredavik et al 2000; Mayo et al 2000). Unfortunately this type of extended services is non-existent in under-resourced rehabilitation settings such as in the Western Cape.

Post 1994 a national health care policy was promulgated and adopted in South Africa, in support of primary health care with implications for the nature of treatments offered and where they are located. In the primary health care approach, Community Health Centres (CHCs) form part of primary level services (A national health plan for South Africa 1994). It is important to note that CHCs have been designated as low intensity facilities in which rehabilitation should be offered by at least one rehabilitation professional or auxiliary rehabilitation worker (Department of Health 2007). The reality of the situation is that $\mathrm{CHCs}$ are tasked with providing rehabilitation services to acute stroke patients. Hale and Wallner (1996) asserted that in South Africa patients are often discharged despite findings indicating that community rehabilitation services are inadequate for the management of acute and sub-acute patients. These patients often need assistance with performing activities needed to function within their homes and communities. In situations where resources and services are limited and acute patients with stroke live at home and receive out-patient services, it is important to determine the exact needs of the patients. In addition, little is known about the outcomes of patients with stroke who receive rehabilitation at out-patient centres in this context. The aim of the study was therefore to determine the activity limitations of patients with stroke receiving out-patient rehabilitation at CHCs in the Western Cape, South Africa. This information could be used to implement stroke rehabilitation services that are appropriate for underresourced countries.

\section{METHODS}

Ethical approval for the study was obtained from the relevant Institutional Review Board. A longitudinal, observational study was used to collect the data relating to the outcomes of stroke rehabilitation at CHCs. All patients who were consecutively admitted to the centres for therapy and who met specific inclusion criteria were included in the study. The inclusion criteria included a first-ever stroke as defined by the World Health Organisation (1989) patients who had suffered a previous stroke were excluded as they could have impairments as a result of the previous stroke, the study also included those who had Rivermead Motor Assessment (RMA) scores of: Gross function (RMA-G) $\leq 11$; and/or Leg and trunk function $($ RMA-LT) $\leq 8$; and/or Arm function (RMA-A) $\leq 12$. This meant that the participants still had some degree of motor impairment (Lincoln and Leadbitter, 1979), aged between $<35$ and $>85$ years. Patients were excluded if they had other neurological impairments with permanent damage, such as a previous head injury or spinal cord injuries, if they had stroke-like symptoms due to sub-dural haematoma, brain tumour, encephalitis or trauma, if their stroke had occurred more than six weeks before, a pre-stroke Barthel Index Score of $<50$ (determined by completing pre-stroke Barthel Index assessment) and if no informed consent had been obtained from the patient or family. The Gross Motor Rivermead Motor Assessment Scale (Lincoln and Leadbitter 1979), the Barthel Index (Mahoney and Barthel 1965) and the Nottingham Extended Activities (Lincoln and Gladman 1992) were used to measure activity limitations of the participants. The sub-scale of the Rivermead Motor Assessment Scale provides information about gross motor function, while the Barthel Index provides information about basic activities of daily living and the Nottingham Extended Activities of Daily Living Scale provides information about extended activities of daily living. All the instruments used were valid and reliable (Finch et al 2002). Although the validity and reliability testing of the instruments had not been conducted in the local setting the items tested were appropriate to the local populations and had been used successfully in other studies conducted in South Africa (Joseph and Rhoda 2011). As a non- probability sampling method was used the use of regression analysis guided the determination of sample size. When conducting regression analysis the literature recommends that for every one dependent variable at least ten independent variables are needed (Munro 2001). The researcher identified eight specific variables from the literature (Meijer et al 2003) that predicted motor and functional outcome post-stroke. Thus, a total of 80 participants would be sufficient. As the study was longitudinal in nature the researcher had to plan for dealing with attrition due to drop-out and therefore decided to recruit 100 participants which would be sufficient for the statistical analysis anticipated and to address drop-out effect.

To access the participants, the researcher contacted the CHCs thrice a week for the names of patients who had suffered a first-ever stroke and were newly admitted to the centres. Once the names and contact details of eligible patients were obtained they were contacted and an appointment was set up either at the participants' home or at the CHC. In cases where telephone numbers were not available, the researcher or research assistant went to the patient's home. The aim of the study was explained and the patients were invited to participate in the study. If the patient was eligible to be included in the study, the necessary baseline questionnaires were completed once written informed consent was obtained. Once the researcher or research assistant had finished collecting the baseline data, the participants were informed that they would be contacted for an appointment for the two and six month follow-up assessments. As was the case with the baseline data, the data was collected at a place that was convenient for the participant which was either their home or the physiotherapy department at the CHC.

The data were analysed using the Statistical Package for Social Science version 15 and 16 (SPSS) and Statistical Analysis System(SAS). To ensure correct capturing, the data were entered twice and compared using the COMPARE procedure in SAS. The Shapiro Wilk W test was used to assess normality of the 


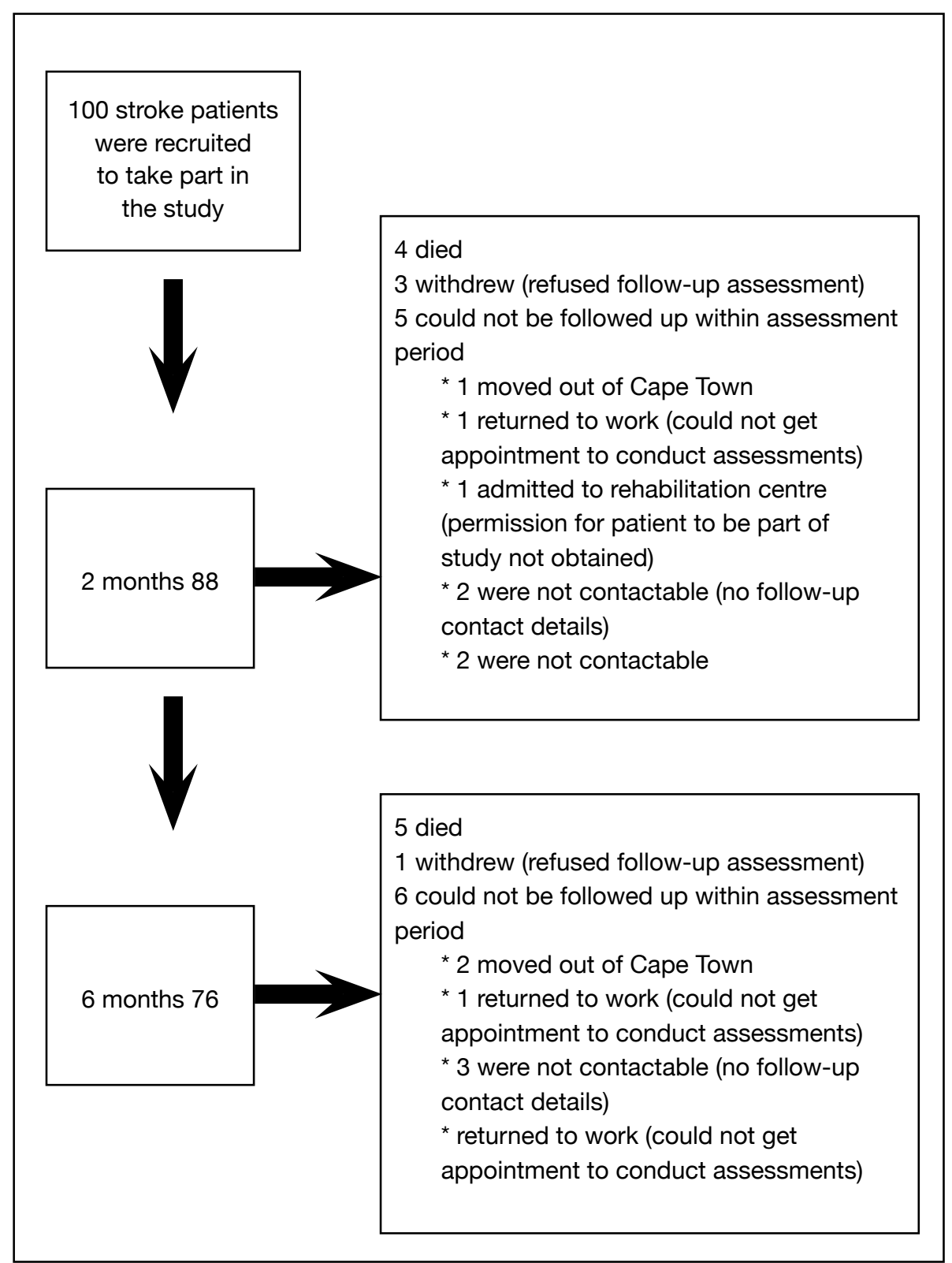

Figure 1: Results of participant recruitment data. Both descriptive and inferential statistics were used to analyse the data. Frequencies of the different socio-demographic and activity limitations were determined. These frequencies related to data collected at baseline, two months and six months post-stroke where appropriate. An intention-to-treat analysis was adopted in which a mixed effects regression model was used to assess for significant differences on the outcome measures between the different assessment periods at alpha level of 0.05 . This model allows for the inclusion of all cases in the analysis irrespective of the loss to follow-up.

\section{RESULTS}

\section{Participant recruitment}

A total of 100 participants were recruited into the study from June 2005 to November 2008. Twelve participants dropped out of the study at the two month assessment period and a further 12 dropped out at six month assessment period. Figure 1 presents the results of participants recruited into the study.

\section{Description of the participants}

The study sample consisted of an equal number of males $50(50 \%)$ and females $50(50 \%)$. The mean age of the population was 61.0 with a standard deviation of 10.55 and ages ranged from 36 years to 85 years. The participants were recruited into the study at a median 21 days post stroke.

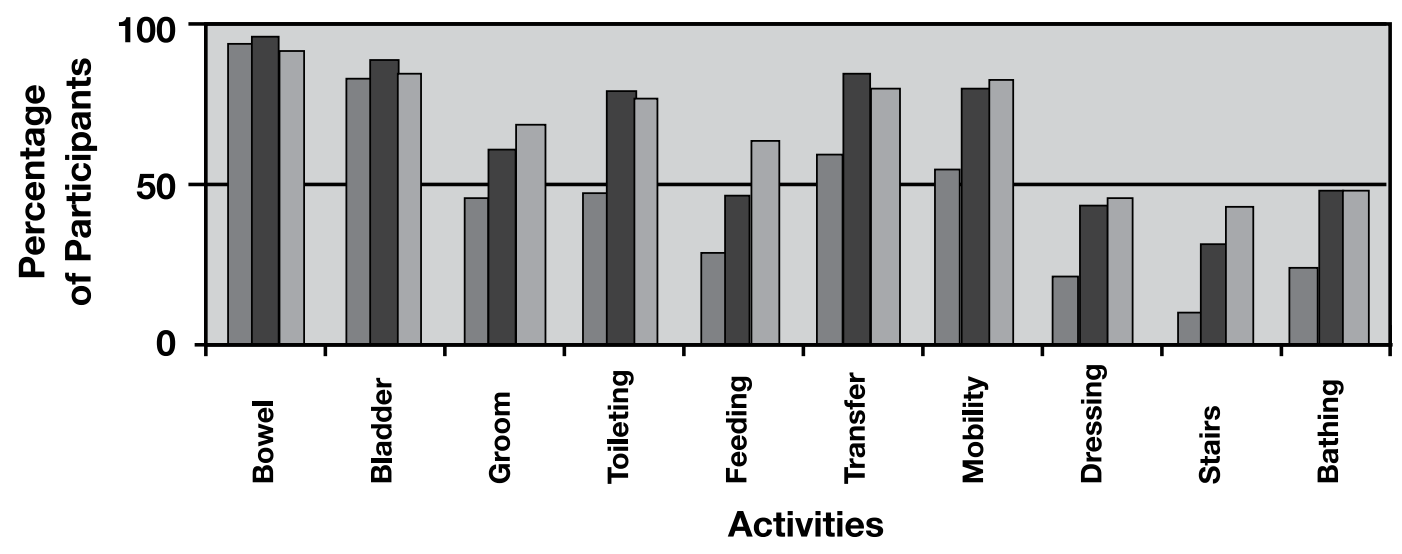


Activity Limitations of the participants Gross Motor Function as measured by RMA-G

With regards to gross motor function, unsupported sitting (item 1) was the activity that the majority of the participants were able to perform independently. In sharp contrast, the majority of the participants could not run (item 12) and hop (item 13) at baseline, two and six months post stroke. An increase in the number of participants who were able to perform various other activities occurred mainly between baseline and two months post onset stroke (Table 1).

\section{Basic activities of daily living as} measured by Barthel Index

At baseline the majority of the participants needed assistance with all basic activities of daily living except bowel, bladder, transfers and mobility. At the two month follow-up period more patients became independent. Nevertheless still more than 50 percent needed assistance with feeding, dressing, stair climbing and bathing. At six month the majority of patients still needed assistance with dressing, stair climbing and bathing (Figure 2).

\section{Barthel Index Scores according to level of severity}

The participants' ability to perform functional activities of daily living was measured using the Barthel Index (Table 2). The results indicated that at the 6 month assessment period only $19.7 \%$ of the participants were fully independent with the majority (80.3\%) still needing assistance with at least one activity of daily living.

\section{Instrumental Activities of daily living as measured by NEADL.}

Participants improved their ability to perform the majority of activities independently between the two and six months assessment periods. As is presented in table 3 , at six months post stroke the vast majority of patients could not write letters independently $(92.1 \%)$ or drive a car (96.1\%). The majority of the participants were also not able to climb stairs, travel on public transport, wash dishes, do the washing, house-
Table 1: Percentage of participants who were independent on the 13 sub-items of the RMA-G at baseline $(n=100)$, two $(n=88)$ and six months $(n=76)$ post stroke.

\begin{tabular}{|l|l|l|l|}
\hline RMA-G Items & Baseline & Two months & Six months \\
\hline Unsupported sitting & 93.4 & 98.7 & 97.4 \\
\hline Lying to sitting & 92.1 & 94.7 & 94.7 \\
\hline Sitting to standing & 84.2 & 89.5 & 88.2 \\
\hline $\begin{array}{l}\text { Transfer to wheelchair } \\
\text { towards unaffected side }\end{array}$ & 82.9 & 88.2 & 88.2 \\
\hline $\begin{array}{l}\text { Transfer to wheelchair } \\
\text { towards affected side }\end{array}$ & 85.3 & 88.2 & 86.8 \\
\hline $\begin{array}{l}\text { Walk indoors 10m with } \\
\text { an aid }\end{array}$ & 75.7 & 85.5 & 82.9 \\
\hline $\begin{array}{l}\text { Climb stair independ- } \\
\text { ently }\end{array}$ & 39.5 & 60.5 & 65.8 \\
\hline $\begin{array}{l}\text { Walk 10m indoors } \\
\text { without aid }\end{array}$ & 58.9 & 75.0 & 78.9 \\
\hline $\begin{array}{l}\text { Pick up bean bag } \\
\text { from floor }\end{array}$ & 69.1 & 80.3 & 6.9 \\
\hline $\begin{array}{l}\text { Walk outside 40m } \\
\text { W steps }\end{array}$ & 41.7 & 3.9 & 5.3 \\
\hline $\begin{array}{l}\text { Run 10m } \\
\text { Hop on affected leg }\end{array}$ & 2.8 & 6.8 & 6.8 \\
\hline
\end{tabular}

Table 2: Barthel Index Score according to participants' severity for each assessment point.

\begin{tabular}{|l|l|l|l|}
\hline Category & \multicolumn{3}{|c|}{ Number (\%) of participants } \\
\hline & $\begin{array}{l}\text { Baseline } \\
\mathrm{n}=100\end{array}$ & $\begin{array}{l}\text { 2 months } \\
\mathrm{n}=88\end{array}$ & $\begin{array}{l}6 \text { months } \\
\mathrm{n}=76\end{array}$ \\
\hline $\begin{array}{l}\text { Dependent } \\
(0-55)\end{array}$ & $41(41.0)$ & $17(19.3)$ & $13(17.1)$ \\
\hline $\begin{array}{l}\text { Moderate } \\
\text { Assistance } \\
(60-80)\end{array}$ & $36(36.0)$ & $27(30.7)$ & $17(22.4)$ \\
\hline $\begin{array}{l}\text { Minimal } \\
\text { Assistance } \\
(85-95)\end{array}$ & $21(21.0)$ & $33(37.5)$ & $31(40.8)$ \\
\hline $\begin{array}{l}\text { Independent } \\
(100)\end{array}$ & $2(2.0)$ & $11(12.5)$ & $15(19.7)$ \\
\hline
\end{tabular}


Table: 3. Number (\%) of participants independent in items of the NEADL at two months and six months post stroke onset.

\begin{tabular}{|c|c|c|}
\hline \multirow[t]{2}{*}{ Variable } & \multicolumn{2}{|c|}{ Participants n(\%) } \\
\hline & $\begin{array}{c}2 \text { months } \\
n=88\end{array}$ & $\begin{array}{c}6 \text { months } \\
n=76\end{array}$ \\
\hline \multicolumn{3}{|l|}{ Mobility } \\
\hline Walk outside & $66(75.0)$ & $59(77.6)$ \\
\hline Climb stairs & $32(36.4)$ & $34(44.7)$ \\
\hline Get in and out of car & $55(62.5)$ & $49(64.5)$ \\
\hline Walk over uneven ground & $47(53.4)$ & $50(65.8)$ \\
\hline Cross roads & $46(52.3)$ & 48 (63.2) \\
\hline Travel on public transport & $16(18.2)$ & $25(32.9)$ \\
\hline \multicolumn{3}{|l|}{ Kitchen Activities } \\
\hline Make yourself a hot drink & $47(53.4)$ & $49(64.5)$ \\
\hline Take hot drinks from one & $48(54.5)$ & $50(65.8)$ \\
\hline \multicolumn{3}{|l|}{ room to another } \\
\hline Do the washing up & $32(36.4)$ & $32(42.1)$ \\
\hline Make yourself a hot snack & $33(37.5)$ & 39 (51.3) \\
\hline \multicolumn{3}{|l|}{ Domestic Tasks } \\
\hline Manage your own money when out & 47 (53.4.) & $46(60.5)$ \\
\hline Wash small items of clothing & $30(34.1)$ & $34(44.7)$ \\
\hline Do your own housework & $23(26.1)$ & $26(34.2)$ \\
\hline Do your own shopping & $17(19.3$ & $23(30.3)$ \\
\hline Do a full clothes wash & $8(9.1)$ & $12(15.8)$ \\
\hline \multicolumn{3}{|l|}{ Leisure activities } \\
\hline Read newspaper and books & $54(61.4)$ & $48(63.2)$ \\
\hline Use the telephone & $44(50.0)$ & $48(63.2)$ \\
\hline Write letters & $4(4.5)$ & $6(7.9)$ \\
\hline Go out socially & $19(21.6)$ & $23(30.3)$ \\
\hline Manage your own garden & $11(12.5)$ & $17(22.4)$ \\
\hline Drive a car & $6(6.8)$ & $3(3.9)$ \\
\hline
\end{tabular}

Table 4 Time effects: Changes in activity limitations as measured by the RMA-G, Barthel Index and EADL for different assessment periods

\begin{tabular}{|l|l|l|l|}
\hline $\begin{array}{l}\text { Assessment } \\
\text { period }\end{array}$ & $\begin{array}{l}\text { RMA-G } \\
\text { LS mean (CI) }\end{array}$ & $\begin{array}{l}\text { Barthel Index } \\
\text { LS mean(CI) }\end{array}$ & $\begin{array}{l}\text { NEADL } \\
\text { LS mean(CI) }\end{array}$ \\
\hline $\begin{array}{l}\text { Baseline }-2 \\
\text { months }\end{array}$ & $1.23(0.89 ; 1.58)^{\star}$ & $12.5(9.24 ; 15.8)^{\star}$ & \\
\hline $2-6$ months & $0.17(-0.25 ; 0.6)$ & $0.45(-3.19: 4.09)$ & $1.33(0.577 ; 2.076)^{\star}$ \\
\hline $\begin{array}{l}\text { Baseline }-6 \\
\text { months }\end{array}$ & $1.40(0.92 ; 1.88)^{\star}$ & $13.0(8.74-17.2)^{\star}$ & \\
\hline
\end{tabular}

hold and shopping, go out socially and manage their garden.

\section{Change of scores across time points}

From the results of the mixed effects regression model which is presented in terms of LS means and (CI) it becomes evident that the difference scores observed between baseline and two months, as well as between baseline and six months were found to be significant for both the RMA-G and the Barthel Index Scores. However, no significant changes were noted between two and six months for both outcome measures. The difference scores observed between two months and six months, for the NEADL scores were also found to be significant. These results are presented in table 4 .

\section{DISCUSSION}

Limitations in activities are a major challenge to patients who have experienced a stroke. The individual activities the participants could not perform at baseline meant that they needed someone to care for them probably on an almost full-time basis. As a result they often experience feelings of helplessness and frustration (Dowswell et al 2000). Although the majority of the participants only needed minimal assistance (Granger et al 1979), at six months the activities that they could still not perform independently such as dressing, stair climbing and bathing once again highlighted their dependence on carers. These activities which stroke patients have difficulty executing are similar to those reported locally (Rouillard 2006) and internationally (Hartman-Maier et al 2007). Access to homes or public buildings which have stairs would also be difficult for these patients as they could not climb stairs independently.

The fact that more than $50 \%$ of the participants still needed assistance with climbing stairs, travelling using public transport, doing housework or managing the garden at six months could be as a result of environmental barriers such as bathrooms that are inaccessible, or homes that have steps at front doors. This means that in patients where improvement in functional activity is no longer expected, the rehabilitation inter- 
ventions should therefore be focused on changing the environment of the person with the disability (Helander 1999). The building of ramps would be needed to assist where patients are not able to climb stairs and the provisions of assistive devices to assist with bathing.

The inability to perform certain tasks that they could do previously, such as household tasks and shopping, meant that these tasks had to be performed by others, thus resulting in a role change. A change of role results in patients with stroke experiencing a sense of losing control (Dowswell et al 2000). The participants' inability to access public transport was a major concern. Only one out of three participants in the present study was able to use public transport. The socio-economic status of the study population with $80.7 \%$ on an income below R1000 a month implies that only a small number of the participants owned a car. The ability to use public transport is therefore important. A lack of access to transport could impact on other domains of the individual's life such as involvement in recreational activities. Their visits outside of the house sometimes become limited to follow-up medical appointments with the doctor (Rochette et al 2007).

It should however be noted that the inability to use public transport could have been intrinsic to the patient, meaning as a result of motor or other impairments or as a result of extrinsic factors such as accessibility of the transport system. Accessibility of public transport was however not investigated in this study and should be explored in future research.

The pattern of neurological recovery that occurred in the participants is in line with what was recorded in the literature. A number of studies have indicated that neurological recovery occurs early after stroke (Duncan et al 2000; Jørgensen et al 1995). Jørgensen et al (1995) reported that in patients who were part of a Copenhagen study those who had a severe stroke reached their maximum recovery within 15 weeks. For the participants classified as having a mild stroke in the Copenhagen study, neurological recovery was reached within six weeks post-stroke onset. As is reported elsewhere (Rhoda 2010), the majority of the patients in the present study were classified as having a mild stroke as measured by the NIHSS (Brott et al 1989). One could conclude that the lack of significant neurological recovery between two and six months could be related to the fact that the participants had reached their best measurable level of neurological recovery (Jørgensen et al 1995). Duncan et al (2000) also reported that the most evident recovery of neurological impairments occurs within the first month, although some neurological recovery could still be observed for up to six months in patients with severe stroke. When comparing the results of the study with that of the Copenhagen study (Jørgensen et al 1995) it should be noted that the severity of stroke was determined using different scales when comparing results of the present study with those of studies conducted previously and results should therefore be compared with caution. However, the finding that neurological and motor recovery occur earlier in the post-stroke period appears to be robust based on its consistency despite the different scales being used.

The participants could be classified as having moderate disability (Jørgensen et al 1995). Jørgensen et al (1995) found that patients with moderate disability attained their best levels of functioning within seven weeks. Although this is the case the majority $(80.3 \%)$ of the participants in the current study still needed assistance with at least one activity of daily living at six months post stroke with only $19.7 \%$ being totally independent with a Barthel score equalling 100. In contrast Jørgensen et al (1995) reported total independence in $46 \%$ of the participants treated with increased intensity in a stroke unit from acute admission to end of rehabilitation. The lack of a statistically significant improvement between two and six months noted in the present study could, therefore, be due to decreased intensity of treatment received by the participants. The stroke patients attending the CHCs only received therapy on average once a week (Rhoda et al 2009).
Although more than half of the participants were not able to perform certain instrumental activities at six months post stroke, the statistically significant improvement found between the two and six month NEADL scores might be related to improved coping strategies by some participants to conduct certain activities. It could be that at the early stages post stroke patients tend to do less and are more dependent on others to perform activities. After realizing that certain movements are not going to return or are not returning at the rate they expect they start doing activities for themselves.

Therapists working at CHCs should be aware of the level of neurological and motor impairments of the stroke patients and should subsequently provide interventions that are appropriate and would optimally facilitate recovery. For example, depending on the severity of the stroke experienced, the aim of rehabilitation for patients who are past three to six months post-stroke should not necessarily be to improve neurological or motor impairments but to address factors such as improving activity and participation. This could be done as improvements in activity limitations and participation restrictions could still occur even when no further neurological or motor recovery is expected.

\section{CONCLUSION}

Although the participants had mild disability at six months post stroke and could almost function independently within their homes, their inability to be independent in certain instrumental activities of daily living limited their re-integration into their communities. We recommend that therapists should include activities which could facilitate re-integration into the community in their rehabilitation of patients with stroke. In addition the rehabilitation of stroke patients at the CHCs should include an intersectorial approach. The therapists should liaise with other sectors such as the housing department and the department of transport to address the environmental barriers that are limiting the patients from being independent. 


\section{REFERENCES}

A National Health Plan for South Africa 1994 http://www.bhfglobal.com/files/bhf/Heather\%20 McLeod\%20-\%20ANC\%20HEALTH\%20 PLAN\%201994.pdf. Retrieved 24 February 2010 (a) 15h38:

Anderson C, Rubenach S, Mhurchu C, Clarke M, Spensor C, Winsor A 2000 Home or Hospital for Stroke Rehabilitation? Results of a Randomised Controlled Trial: 1: Health Outcomes at 6 Months. Stroke 31:1024-1031.

Bradshaw, D., Nannan, N., Laubsher, R., Groenewald, P., Joubert, J., Nojilana, B., et al. (2004). South African National Burden of Disease Study 2000. Estimates of provincial mortality, Cape Town: Medical Research Council.

Bautz-Holter E, Sveen U, Rygh J, Rodgers H, Bruun Wyller T 2002 Early supported discharge of patients with acute stroke: a randomized controlled trial. Disability \& Rehabilitation 24(7):348-355.

Bonita R, Broad J, Beaglehole R 1997 Ethnic Differences in Stroke Incidence and Case Fatality in Auckland, New Zealand. Stroke 28:758-761.

Department of Health (DOH), Western Cape Province. (2007). Comprehensive Service Plan for the Implementation of Health Care 2010.

Donnelly M, Power M, Russell M, Fullerton K 2004 Randomised Controlled Trial of an Early Discharge Rehabilitation Service. The Belfast Community Stroke Trial. Stroke 35:127-137.

Dowswell G, Lawler J, Dowswell T, Young J, Forster A, Hearn J 2000 Investigating recovery from stroke: a qualitative study. Journal of Clinical Nursing 9(4):507-515.

Duncan P, Lai S, Keighly J 2000 Defining post-stroke recovery: implications for design and interpretation of drug trials. Neuropharmacology 39:835-841

Feigin VL, Lawes CM, Bennett DA, Anderson CS 2003. Stroke epidemiology: A review of population-based studies of incidence, prevalence, and case-fatality in the late 20th century. Lancet Neurology, 2(1), 43-53.

Finch C, Brooks D, Stratford P, Mayo N 2002 Physical Rehabilitation Outcomes: A Guide to Enhanced Clinical Decision-making. Lippincott Williams \& Wilkins, Canada

Granger C, Dewis L, Peters N, Sherwood C, Barret J 1979 Stroke Rehabilitation: analysis of repeated Barthel Index Measures. Archives of Physical Medicine and Rehabilitation 60:14-17.

Hale L, Wallner P 1996 The Challenge of Service Provision in South Africa for Patients with hemiplegia. Physiotherapy 62(3):156-158.

Hartman-Maeir A, Soroker N, Ring H, Avni N, Katz N 2007 Activities, participants and satisfaction one-year post stroke. Disability and Rehabilitation 29(7):559-566.
Helander E 1999 Prejudice and Dignity - An Introduction to Community-Based Rehabilitation, Second Ed. United Nations Development Programme, New York

Indredavik B, Fjaertoft H, Ekeberg G, Loge A, Morch B 2000 Benefit of an Extended Stroke Unit Service with Early Supported Discharge. A Randomised Controlled Trial. Stroke 31:29892994.

Joseph C, Rhoda A 2011 Outcome Measures used to assess disability post stroke within the framework of the ICF: A literature Review. Journal of Community and Health Sciences 6(1): 39-52

Jørgensen H, Nakayama $H$, Raaschou $H$, Olsen T 1995 Recovery of walking function in stroke patients: The Copenhagen Stroke Study. Archive of Physical Medicine \& Rehabilitation 76(1):27-32.

Kwakkel G, Kollen B, Lindeman E 2004 Understanding the pattern of Functional Recovery after Stroke: Facts and Theories. Restorative Neurology and Neuroscience 22:281-299.

Lincoln N, Gladman J 1992 The Extended Activities of Daily Living Scale: A Further Validation. Disability and Rehabilitation 14: 41-43.

Lincoln N, Leadbitter D 1979 Assessment of Motor Function in Stroke Patients. Physiotherapy 65(2):48-51.

Mahoney F I, Barthel D 1965 Functional evaluation: the Barthel Index. Maryland State Medical Journal 14:56-61.

Mayo N, Wood-Dauphinee S, Cote R, Gayton D, Carlton J, Buttery J, Tamblyn R 2000 There's No Place Like Home: An Evaluation of Early Supported Discharge for Stroke. Stroke 31: 1016-1023.

Mayo N, Wood-Dauphinee S, Ahmed S, Gordon C, Higgins J, McEwen S, Salbach N 1999 Disablement following stroke. Disability and Rehabilitation 21(5/6):258-268.

Meijer R, Ihnenfeldt D, de Groot I, van Liembeek J, Vermeulen M, de Haan R 2003. Prognostic factors for ambulation and activities of daily living in the subacute phase after stroke. A systematic review of literature. Clinical Rehabilitation 17, 119-129.

Munro B H 2001 Statistical Methods for Health Care Research. Philadelphia, New York, Baltimore: Lippincott.

Rhoda A 2010 The Rehabilitation of Stroke Patients at Community Health Centres in the Metropole Region of the Western Cape. PhD Thesis University of the Western Cape. Cape Town. South Africa.

Rhoda A, Hendry J 2006 Rehabilitation of Stroke Patients treated at a community based rehabilitation centre. Journal of Community and Health Sciences 1(1):47-53.
Rhoda A, Mpofu R, DeWeerdt W 2009 The Rehabilitation of stroke patients in the Western Cape. The South African Journal of Physiotherapy 65(3):3-7.

Rochette A, Desrosiers J, Bravo G, St-Cyr/ Tribble D, Bourget A 2007 Changes in participation after a mild stroke: quantitative and qualitative perspectives. Topics in Stroke Rehabilitation 14(3) 59-69.

Rouillard S 2006 Pattern of recovery and Outcome after Stroke in Patients Accessing a Western Cape Rehabilitation facility. Unpublished Master's thesis University of Cape. Cape Town. South Africa.

SASPI Project Team 2004. Prevalence of Stroke Survivors in Rural South Africa: Results from the Southern African Stroke Prevention Initiative (SASPI) Agincourt Field Site. Stroke 36:627-632.

Wade T W, de Jong B 2000 Recent advances in rehabilitation. British Medical Journal 320: 1385-1388

Walker R, Mclarty D, Masuki G, Kitange, H, Whiting D, Moshi A, Massawe J, Amaro R, Mhina A, Alberti K 2000 Age Specific Prevalence of impairment and disability relating to hemiplegic stroke in the Hai District of Northern Tanzania. Journal of Neurology Neurosurgery and Psychiatry 68:744-749.

World Health Organisation 2001. International Classification of Functioning, Disability and Health: ICF short version: WHO Library Cataloguing-in-Publication Data.

World Health Organisation 1989. Recommendations on Stroke Prevention, Diagnosis and Therapy. Report of the WHO Task Force on Stroke and other Cerebrovascular Disorders. 